\title{
Estudo da fração inspirada de oxigênio na isquemia-reperfusão pulmonar em ratos $^{1}$
}

\author{
Study of ventilation with different inspired oxygen concentration on lung ischaemia- \\ reperfusion injury in rats
}

\section{Rafael José Silveira' ${ }^{2}$ Fábio May da Silva ${ }^{3}$, Danilo Wilhelm Filho ${ }^{4}$, João José de Deus Cardoso ${ }^{5}$, Luiz Eduardo Villaça Leão ${ }^{6}$}

1. Trabalho realizado na Universidade Federal de Santa Catarina (UFSC).

2. Mestre em Ciências Médicas pela UFSC, Cirurgião Torácico da Secretaria Estadual de Saúde de Santa Catarina.

3. Doutor em Cirurgia Torácica pela Universidade Federal de São Paulo - Escola Paulista de Medicina (UNIFESPEPM), Professor de Cirurgia da Universidade do Sul de Santa Catarina.

4. Professor Titular do Departamento de Ecologia e Zoologia da UFSC.

5. Professor Adjunto, Doutor, Chefe do Serviço de Cirurgia Torácica e do Departamento de Clínica Cirúrgica da UFSC.

6. Professor Titular da Disciplina de Cirurgia Torácica do Departamento de Cirurgia da UNIFESP-EPM.

\section{RESUMO}

OBJETIVO: Estudar o efeito das frações inspiradas de oxigênio $\left(\mathrm{FiO}_{2}\right)$ a 0,21, 0,40 e 1,00 na isquemia-reperfusão pulmonar.

MÉTODOS: Foram utilizados 40 ratos Wistar, distribuídos aleatoriamente em quatro grupos. O grupo I foi o controle e, nos grupos II, III e IV, os animais foram ventilados durante a isquemia-reperfusão com $\mathrm{FiO}_{2}$ a 0,21, 0,40 e 1,00 respectivamente. O modelo utilizado foi de isquemia-reperfusão normotérmica, in situ. O tempo de isquemia foi de 30 minutos e o de reperfusão, de 10 minutos. Como parâmetros de avaliação, utilizou-se a pressão arterial média sistêmica (PAM), a relação entre a pressão parcial de oxigênio e a fração inspirada de oxigênio $\left(\mathrm{PO}_{2} / \mathrm{FiO}_{2}\right)$, a dosagem da glutationa reduzida (GSH) e das substâncias reativas ao ácido tiobarbitúrico (TBARS) no tecido pulmonar e a relação entre o peso pulmonar úmido e o peso pulmonar seco.

RESULTADOS: Os resultados mostraram que a ventilação com $\mathrm{FiO}_{2}$ a 0,21 , quando comparada à ventilação com $\mathrm{FiO}_{2}$ a 0,40 e 1,00, durante o período de isquemia-reperfusão, apresentou menor diminuição da PAM, melhor relação $\mathrm{PO}_{2} / \mathrm{FiO}_{2}$, maior valor na medida da $\mathrm{GSH}$, menor produção das TBARS e menor formação de edema pulmonar.

CONCLUSÃO: A ventilação com baixa $\mathrm{FiO}_{2}(0,21)$ mostrou melhores resultados quando comparada àquelas realizadas com $\mathrm{FiO}_{2}$ mais elevadas $(0,40$ e 1,00$)$ na isquemia-reperfusão pulmonar.

DESCRITORES: Transplante de pulmão. Preservação de órgãos. Reperfusão. Radicais livres. Ratos.

\section{ABSTRACT}

PURPOSE: To evaluate the $\mathrm{FiO}_{2}$ effect at $0,21,0,40$ and 1,00 on the lung ischaemia-reperfusion injury.

METHODS: Forty Wistar rats were randomly allocated in 4 groups. The group I was the control one, and in groups II, III, IV rats were ventilated during the ischaemia-reperfusion at $0,21,0,40$ and 1.00 
$\mathrm{FiO}_{2}$ respectively. The ischaemia time was 30 minutes and the reperfusion time was 10 minutes. The model used was normothermic ischaemia-reperfusion, in situ. As assessment parameters, the systemic average arterial pressure (PAM), the oxygen arterial partial pressure/ inspired oxygen fraction relation $\left(\mathrm{PO}_{2} / \mathrm{FiO}_{2}\right)$, the level of reduced glutathione $(\mathrm{GSH})$ and barbituric acid substances reactive measure (TBARS) in the lung tissue and the lung wet/dry weight ratio were used.

RESULTS: The results showed that the ventilation with $\mathrm{FiO}_{2}$ at 0,21 , when compared with the ventilation at 0,40 and $1,00 \mathrm{FiO}_{2}$ during the ischaemia-reperfusion period, presented lower PAM, better $\mathrm{PO}_{2} / \mathrm{FiO}_{2}$ relation, higher values in the reduced glutathione measures, lower production of the TBARS and lower formation of lung edema.

CONCLUSION: The ventilation with low $\mathrm{FiO}_{2}(0.21)$ showed better results when compared to those performed with higher $\mathrm{FiO}_{2}(0,40$ and 1,00$)$ on the lung ischaemia-reperfusion injury.

KEY WORDS: Lung transplantation. Organ preservation. Reperfusion. Oxygen free radicals. Rats.

\section{Introdução}

A realização do primeiro transplante pulmonar com sucesso, em 1983, tornou esse procedimento uma opção para o tratamento de pacientes com doenças terminais do pulmão ${ }^{1}$.

No entanto, fatores como as dificuldades na obtenção do enxerto pulmonar, a rejeição, as infecções pós-operatórias e as lesões decorrentes da preservação do órgão têm dificultado essa modalidade terapêutica na prática clínica ${ }^{2}$.

Para uma boa evolução pós-operatória, é fundamental que se diminua a lesão decorrente do período entre a retirada do órgão do doador e a sua respectiva implantação no receptor. Essa lesão é conhecida como "lesão da isquemia-reperfusão", cujo mecanismo baseia-se, fundamentalmente, na formação e liberação de espécies reativas de oxigênio ${ }^{3}$ (EROs).

A lesão da isquemia-reperfusão pode determinar a ocorrência de infiltrado pulmonar, hipoxemia arterial, diminuição da complacência pulmonar e aumento da resistência vascular pulmonar.

Essas manifestações ocorrem no período pósoperatório imediato ao transplante do pulmão e são conhecidas como resposta de reimplante pulmonar que, dependendo da intensidade, pode evoluir para a falência primária do enxerto pulmonar e a morte do receptor ${ }^{3}$.

Nesse sentido, qualquer medida que diminua a lesão da isquemia-reperfusão estará contribuindo para a melhoria da preservação e, conseqüentemente, do sucesso do transplante pulmonar.

Entre essas medidas, as mais importantes são a hipotermia, o uso de soluções de preservação, vasodilatadores e o padrão ventilatório ${ }^{4}$.

No estudo do padrão ventilatório, destacamse o estado da expansão pulmonar e a concentração alveolar de oxigênio, utilizados durante o período de isquemia-reperfusão.

Em relação ao estado da expansão pulmonar, a insuflação é superior à atelectasia. A insuflação mantém a distensão alveolar, estimula a secreção de surfactante, melhora a distribuição da solução de preservação e diminui a pressão de reperfusão 5,6 .

A manutenção da ventilação, durante a isquemia, também parece acrescentar benefícios à preservação. Ulicny e col. ${ }^{7}$ mostraram que a ventilação com oxigênio diminuía a lesão decorrente da isquemia-reperfusão e sugeriram que esse fato devia-se a uma oferta constante de oxigênio para o metabolismo celular.

No nosso meio, Cardoso ${ }^{8}$ mostrou que a ventilação, quando comparada à insuflação, num modelo de isquemia-reperfusão normotérmica em ratos, mantém maiores níveis de $\mathrm{PO}_{2}$ e menores níveis de lactato tecidual pulmonar.

A insuflação pulmonar ou ventilação, durante a isquemia, manteria os alvéolos distendidos, o que permitiria a difusão do oxigênio alveolar aos tecidos adjacentes, mantendo o metabolismo celular aeróbio e, conseqüentemente, retardaria ou inibiria o aparecimento do metabolismo anaeróbio. Desse modo, ocorreria uma menor formação de EROs, com melhora da resposta tecidual à 
isquemia-reperfusão ${ }^{9}$.

A concentração alveolar de oxigênio, que apresenta relação com a fração inspirada de oxigênio, durante o período de isquemiareperfusão, não está perfeitamente determinada e permanece um assunto polêmico na literatura.

Koyama e col. ${ }^{10}$ observaram que a ventilação com nitrogênio (oxigênio a $0 \%$ ), durante a isquemia, resulta numa menor lesão da isquemiareperfusão quando comparada à ventilação com oxigênio a $21 \%$ ou $100 \%$.

Van Raemdonck e col. ${ }^{11}$ mostraram que a ventilação com oxigênio a $21 \%$ apresenta maiores níveis de ATP e menores níveis de hipoxantina, quando comparada à ventilação com oxigênio a $100 \%$.

Hamvas e col. ${ }^{12}$ mostraram que pulmões insuflados ou ventilados com oxigênio a 40\%, quando comparados com pulmões insuflados ou ventilados com nitrogênio, durante o período de isquemia, apresentam melhor função pulmonar pós-reperfusão.

Hennington e col. ${ }^{13}$ referiram melhores resultados utilizando a ventilação com oxigênio a $100 \%$ em relação à ventilação com oxigênio a $21 \%$, num modelo de isquemia pulmonar normotérmica em cães.

Por outro lado, Zhao e col. ${ }^{14}$ mostraram um aumento da lesão da isquemia-reperfusão em pulmões ventilados com oxigênio a $100 \%$ e relacionaram esse fato a uma maior liberação de EROs.

Considerando-se esses aspectos polêmicos, o presente estudo teve como objetivo avaliar a ventilação com as frações inspiradas de oxigênio a $0,21,0,40$ e 1,00 na isquemia-reperfusão pulmonar.

\section{Métodos}

\section{Amostra}

Foram utilizados 40 ratos (Rattus norvegicus albinus) da linhagem Wistar, machos, adultos, com peso individual entre 300 e 350 gramas (g), procedentes do Biotério Central da Universidade Federal de Santa Catarina (UFSC).

O experimento foi realizado no Laboratório de Técnica Operatória e Cirurgia Experimental do Departamento de Clínica Cirúrgica da UFSC, depois de o projeto ter sido aprovado pela Comissão de Ética no Uso de Animais em Pesquisa.

Os animais passaram por um período mínimo de adaptação de oito dias, recebendo água e ração ad libitum até o início do experimento, e foram distribuídos aleatoriamente em quatro grupos, descritos a seguir.

GRUPO I: $(\mathrm{n}=10)$ Animais submetidos à ventilação com oxigênio a $21 \%\left(\mathrm{FiO}_{2}=0,21\right)$ e toracotomia (controle).

GRUPO II: $(\mathrm{n}=10)$ Animais submetidos à ventilação com oxigênio a 21\% $\left(\mathrm{FiO}_{2}=0,21\right)$, toracotomia e pinçamento parcial do hilo pulmonar esquerdo (artéria e veia) com micropinças vasculares. Os pulmões foram ventilados com oxigênio a $21 \%\left(\mathrm{FiO}_{2}=0,21\right)$ durante o período de isquemia-reperfusão.

GRUPO III: $(\mathrm{n}=10)$ Animais submetidos à ventilação com oxigênio a $21 \%\left(\mathrm{FiO}_{2}=0,21\right)$, toracotomia e pinçamento parcial do hilo pulmonar esquerdo (artéria e veia) com micropinças vasculares. Os pulmões foram ventilados com oxigênio a $40 \%\left(\mathrm{FiO}_{2}=0,40\right)$ durante o período de isquemia-reperfusão.

GRUPO IV: $(\mathrm{n}=10)$ Animais submetidos à ventilação com oxigênio a $21 \%\left(\mathrm{FiO}_{2}=0,21\right)$, toracotomia e pinçamento parcial do hilo pulmonar esquerdo (artéria e veia) com micropinças vasculares. Os pulmões foram ventilados com oxigênio a $100 \%\left(\mathrm{FiO}_{2}=1,00\right)$ durante o período de isquemia-reperfusão.

\section{Procedimentos}

Os animais receberam como medicação préanestésica a atropina, na dose de 0,25 miligramas por quilo (mg/kg), por via subcutânea, e foram anestesiados com uma mistura de pentobarbital sódico a 3\% e cloridrato de 2-(2,6-xilidino)-5,6dihidro-4H-1,3-tiazina, na proporção de 10:1. Foram administrados 0,10 mililitros $(\mathrm{ml}) / 100 \mathrm{~g}$ de peso da mistura, por via intramuscular, na face interna da pata traseira. $\mathrm{O}$ animal foi considerado anestesiado quando ocorreu a perda do reflexo córneo-palpebral e a ausência da reação motora pela preensão do coxim adiposo da pata dianteira. 
Realizou-se uma traqueostomia cervical, intubação traqueal com um cateter de polietileno n. ${ }^{\circ}$ 6, e iniciou-se a ventilação mecânica.

A ventilação mecânica foi realizada com o aparelho Harvard - 683, numa freqüência de 80 movimentos respiratórios por minuto e volume de ar corrente, calculado por meio da curva Kleinmann \& Radford.

Efetuou-se uma laparotomia mediana para a cateterização da aorta abdominal, com cateter de silicone $n .^{\circ} 0$, utilizado para a heparinização do animal, medida da pressão arterial média sistêmica (PAM) e coleta de sangue para análise da pressão parcial de oxigênio arterial $\left(\mathrm{PO}_{2}\right)$.

A heparinização sistêmica do animal fez-se por meio da administração de $0,50 \mathrm{ml}$ de uma solução de soro fisiológico (solução de cloreto de sódio a $0,9 \%$ ) contendo $0,02 \mathrm{ml}$ de heparina na concentração de $5.000 \mathrm{U} / \mathrm{ml}$.

Efetuou-se uma toracotomia anterior bilateral com secção transversal do esterno no seu um terço médio. Com auxílio do microscópio de luz D. F. Vasconcelos MC-5 em aumento de oito vezes, utilizando-se técnica microcirúrgica, dissecou-se o hilo pulmonar esquerdo e foram individualizadas a artéria e a veia pulmonar esquerda.

As artérias e as veias pulmonar esquerdas foram mantidas pinçadas nos grupos II, III e IV durante o período de isquemia.

O pulmão esquerdo foi submetido a um período de 30 minutos de isquemia e 10 minutos de reperfusão in situ. A temperatura da sala operatória variou entre 19 e 24 graus Celsius.

Durante o período de isquemia-reperfusão, os pulmões foram ventilados com diferentes frações inspiradas de oxigênio $\left(\mathrm{FiO}_{2}\right)$, conforme o grupo estudado.

No tempo denominado de "finalização do ato operatório”, efetuou-se a irrigação pulmonar com soro fisiológico. Para isso, procedeu-se à ligadura das veias cavas superiores e inferior, secção parcial do arco aórtico, cateterismo do átrio direito e injeção lenta de 10,00 ml de soro fisiológico à temperatura ambiente. Em seguida, foram realizadas as pneumonectomias esquerda e direita, o que resultou na morte do animal.

A representação esquemática dos procedimentos efetuados em cada grupo está representada na Figura 1.

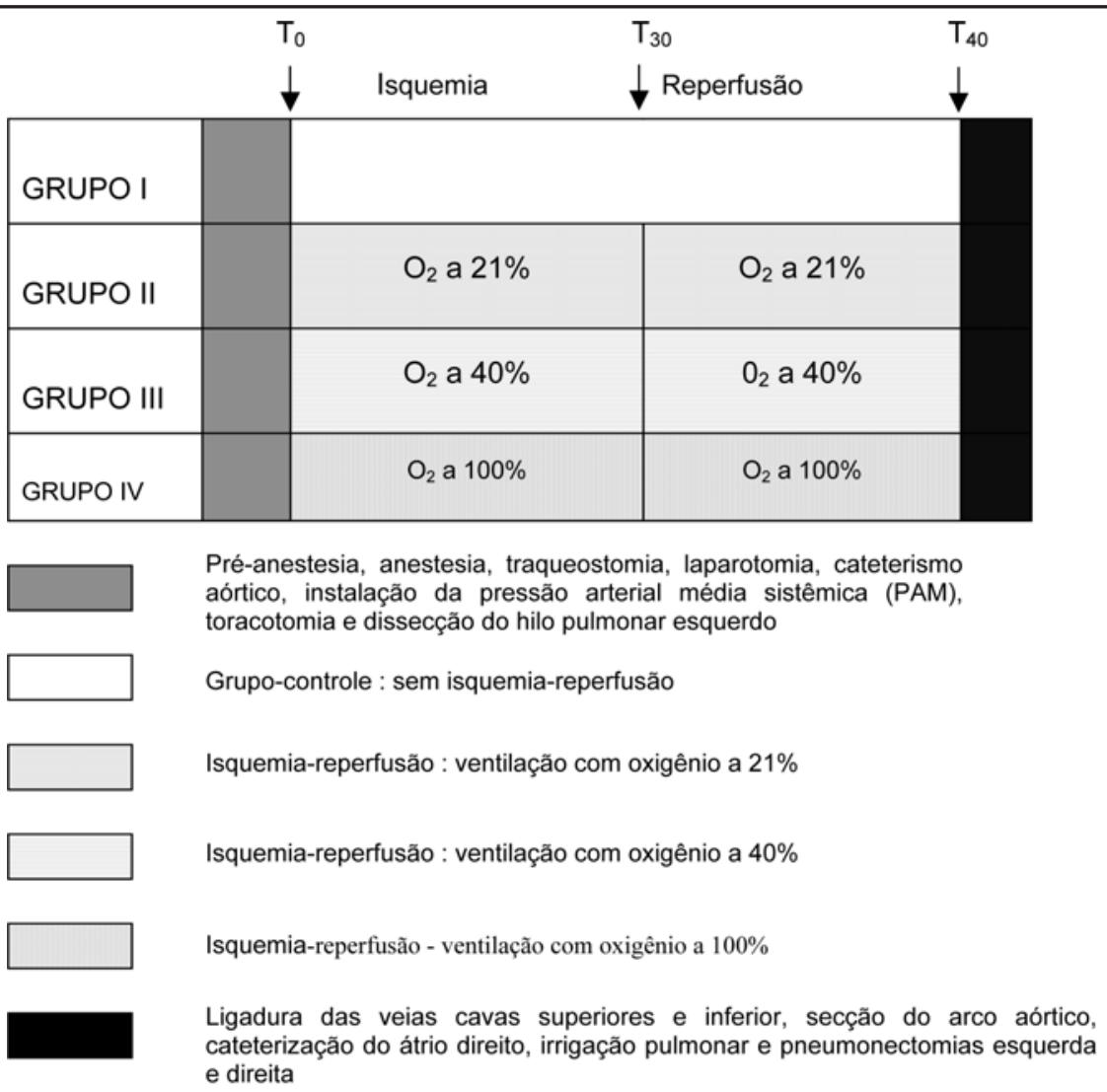

FIGURA 1 - Esquema dos procedimentos trans-operatórios nos diferentes grupos. 
Os métodos de avaliação utilizados foram:

1. Medida da pressão arterial média sistêmica (PAM), realizada nos tempos zero $\left(\mathrm{T}_{0}\right)$ imediatamente antes do pinçamento parcial do hilo pulmonar esquerdo; trinta $\left(\mathrm{T}_{30}\right)$ - 30 minutos após o início da isquemia e imediatamente antes do despinçamento do hilo pulmonar esquerdo; e quarenta $\left(\mathrm{T}_{40}\right)$ - 10 minutos após o início da reperfusão, por meio de um manômetro aneróide, sendo os seus valores expressos em $\mathrm{mmHg}$;

2. Medida da relação entre a pressão parcial de oxigênio arterial e a fração inspirada de oxigênio $\left(\mathrm{PO}_{2} / \mathrm{FiO}_{2}\right)$, realizada nos tempos $\mathrm{T}_{0}, \mathrm{~T}_{30}$ e $\mathrm{T}_{40}$. A medida da $\mathrm{PO}_{2}$ arterial foi realizada por meio de gasometria arterial no Laboratório Central do Hospital Universitário da UFSC;

3. Dosagem da glutationa reduzida (GSH), realizada em amostras de pulmões submetidos à isquemia-reperfusão (pulmão esquerdo) e contralateral (pulmão direito), pelo método de Beutler ${ }^{15}$, sendo os valores expressos em milimóis (mM).

4. Dosagem das substâncias reativas ao ácido tiobarbitúrico (TBARS), realizada em amostras de pulmões submetidos à isquemia-reperfusão e contralateral, por meio de espectrofotometria, sendo os valores expressos em $\mathrm{nmol} \mathrm{g}^{-1}$;

5. Medida da relação entre o peso pulmonar úmido e o peso pulmonar seco, realizada em amostra de pulmão submetido à isquemiareperfusão, por meio da pesagem in natura (peso úmido) e sua relação com a mesma porção após secagem em estufa a $60^{\circ} \mathrm{C}$ por 4 horas (peso seco), utilizando-se uma balança de alta precisão, com os valores expressos em gramas (g).

\section{Análise estatística}

Para análise dos resultados, utilizaram-se os seguintes testes estatísticos:

1 - Análise de variância não paramétrica por postos de Kruskal-Wallis ${ }^{16}$, quando se comparam possíveis diferenças entre os grupos (I x II x III e IV); e
2 - Quando o teste de Kruskal-Wallis apresentou significância estatística, ele foi complementado pelo teste de comparações múltiplas de MANN-WHITNEY ${ }^{16}$ (I x II, I x III, I x IV, II x III, II x IV e III x IV).

3 - Teste de WILCOXON ${ }^{16}$, quando se comparam possíveis diferenças entre os pulmões esquerdo e direito nos quatro grupos (I, II, III e IV)

Quando se compararam possíveis diferenças entre os quatro grupos (I x II x III x IV) no tempo zero, a variável foi usada com o seu valor absoluto.

Quando se compararam possíveis diferenças entre os grupos (I x II x III x IV) no tempo quarenta, preferiu-se utilizar as variáveis:

$\Delta \% 0 \rightarrow 40=\frac{\text { tempo zero }- \text { tempo quarenta }}{\text { tempo zero }}$

Quanto ao sinal, ele foi positivo quando significou um aumento da variável, e negativo quando significou uma diminuição de acordo com o sentido biológico do fenômeno.

Em todos os casos, o nível de rejeição da hipótese de nulidade foi sempre fixado em um nível de confiança de $95 \%$, ou seja, com um valor igual ou menor do que 0,05 (5\%).

Quando os cálculos estatísticos apresentaram um valor significante, usou-se um asterisco $\left(^{*}\right)$ para caracterizá-lo.

As médias foram calculadas apenas a título de informação e serviram para a confecção dos gráficos. 


\section{Resultados}

TABELA 1 - Valores da PAM, nos tempos $\mathrm{T}_{0}, \mathrm{~T}_{30}$ e $\mathrm{T}_{40}$, em mmHg, segundo os grupos.

\begin{tabular}{|c|c|c|c|c|c|c|c|c|c|c|c|c|c|c|}
\hline & \multicolumn{3}{|c|}{ Grupo I } & \multicolumn{4}{|c|}{ Grupo II } & \multicolumn{3}{|c|}{ Grupo III } & \multicolumn{4}{|c|}{ Grupo IV } \\
\hline & 0 & 30 & 40 & & 0 & 30 & 40 & 0 & 30 & 40 & & 0 & 30 & 40 \\
\hline & 110 & 130 & 135 & & 120 & 135 & 135 & 120 & 112 & 102 & & 130 & 100 & 110 \\
\hline & 120 & 116 & 114 & & 115 & 118 & 90 & 118 & 116 & 94 & & 120 & 102 & 110 \\
\hline & 110 & 110 & 115 & & 118 & 117 & 102 & 120 & 110 & 80 & & 130 & 120 & 116 \\
\hline & 120 & 116 & 98 & & 120 & 112 & 110 & 110 & 96 & 70 & & 112 & 104 & 92 \\
\hline & 110 & 102 & 95 & & 115 & 108 & 95 & 110 & 116 & 92 & & 110 & 100 & 88 \\
\hline & 115 & 115 & 90 & & 120 & 100 & 92 & 120 & 90 & 88 & & 115 & 102 & 102 \\
\hline & 115 & 115 & 102 & & 110 & 110 & 108 & 130 & 124 & 110 & & 115 & 100 & 85 \\
\hline & 120 & 108 & 108 & & 120 & 120 & 105 & 110 & 100 & 80 & & 110 & 120 & 120 \\
\hline & 115 & 102 & 102 & & 120 & 120 & 108 & 130 & 110 & 90 & & 115 & 95 & 80 \\
\hline & 115 & 108 & 102 & & 120 & 95 & 95 & 120 & 120 & 108 & & 120 & 90 & 80 \\
\hline Média 115 & 112 & 106 & & 118 & 113 & 104 & 119 & 109 & 91 & & 118 & 103 & 98 & \\
\hline \multicolumn{4}{|c|}{$\begin{array}{c}\text { Kruskal-Wallis }(\mathrm{I} \text { x II x III x IV) } \\
\text { tempo zero }\left(\mathrm{T}_{0}\right)\end{array}$} & \multicolumn{4}{|c|}{$\begin{array}{c}\chi_{\text {crítico }}^{2}=7,82 \\
\chi^{2}=2,40\end{array}$} & \multicolumn{3}{|c|}{$(p=0,49)$} & & & & \\
\hline \multicolumn{4}{|c|}{$\Delta \% 0 \rightarrow 40:$} & \multicolumn{4}{|c|}{$\chi_{\text {calculado }}^{2}=8,80$} & \multicolumn{3}{|c|}{$(\mathrm{p}<0,05)^{*}$} & & & & \\
\hline \multicolumn{4}{|c|}{ Mann-Whitney $(\Delta \% 0 \rightarrow 40)$} & \multicolumn{4}{|c|}{$\mathrm{U}_{\text {crítico }}=23,00$} & & & & & & & \\
\hline \multicolumn{4}{|c|}{ I x II } & \multicolumn{4}{|c|}{$\mathrm{U}_{\text {calculado }}=40,00$} & \multicolumn{3}{|c|}{$(p=0,48)$} & & & & \\
\hline \multicolumn{4}{|c|}{ I x III } & \multicolumn{4}{|c|}{$U_{\text {calculado }}=13,50$} & \multicolumn{3}{|c|}{$(\mathrm{p}<0,05)^{*}$} & & & & \\
\hline \multicolumn{4}{|c|}{ I x IV } & \multicolumn{4}{|c|}{$\mathrm{U}_{\text {calculado }}=31,50$} & \multicolumn{3}{|c|}{$(p=0,16)$} & & & & \\
\hline
\end{tabular}

TABELA 2 - Valores da $\mathrm{PO}_{2} / \mathrm{FiO}_{2}$, nos tempos $\mathrm{T}_{0}, \mathrm{~T}_{30}$ e $\mathrm{T}_{40}$, segundo os grupos.

\begin{tabular}{|c|c|c|c|c|c|c|c|c|c|c|c|c|}
\hline & \multicolumn{3}{|c|}{ Grupo I } & \multicolumn{3}{|c|}{ Grupo II } & \multicolumn{3}{|c|}{ Grupo III } & \multicolumn{3}{|c|}{ Grupo IV } \\
\hline & 0 & 30 & 40 & 0 & 30 & 40 & 0 & 30 & 40 & 0 & 30 & 40 \\
\hline & 334 & 785 & 740 & 671 & 818 & 761 & 418 & 567 & 563 & 413 & 318 & 367 \\
\hline & 538 & 671 & 739 & 709 & 737 & 767 & 436 & 475 & 421 & 609 & 315 & 339 \\
\hline & 439 & 628 & 598 & 666 & 792 & 787 & 357 & 510 & 545 & 429 & 262 & 389 \\
\hline & 528 & 604 & 677 & 533 & 739 & 755 & 809 & 592 & 551 & 547 & 373 & 351 \\
\hline & 642 & 628 & 750 & 590 & 865 & 628 & 652 & 530 & 550 & 552 & 366 & 404 \\
\hline & 334 & 451 & 401 & 547 & 766 & 686 & 623 & 567 & 548 & 519 & 341 & 303 \\
\hline & 823 & 866 & 857 & 490 & 510 & 498 & 542 & 527 & 540 & 761 & 423 & 354 \\
\hline & 723 & 857 & 876 & 408 & 656 & 645 & 633 & 547 & 500 & 407 & 287 & 286 \\
\hline & 528 & 528 & 528 & 593 & 736 & 815 & 500 & 577 & 341 & 610 & 431 & 466 \\
\hline & 647 & 809 & 728 & 583 & 750 & 779 & 545 & 470 & 477 & 435 & 363 & 376 \\
\hline Média & 554 & 683 & 689 & 597 & 737 & 712 & 551 & 590 & 503 & 528 & 347 & 363 \\
\hline \multicolumn{4}{|c|}{$\begin{array}{l}\text { Kruskal-Wallis }(\mathrm{I} \times \mathrm{II} \times \mathrm{III} \times \mathrm{IV}) \\
\text { tempo zero }\left(\mathrm{T}_{0}\right) \text { : } \\
\Delta \% 0 \rightarrow 40:\end{array}$} & \multicolumn{3}{|c|}{$\begin{array}{l}\chi_{\text {crítico }}^{2}=7,82 \\
\chi_{\text {calculado }}^{2}=1,20 \\
\chi_{\text {calculado }}^{2}=24,31\end{array}$} & \multicolumn{3}{|c|}{$\begin{array}{l}(p=0,75) \\
(p<0,05)^{*}\end{array}$} & & & \\
\hline $\begin{array}{r}\text { Mann- } \\
\text { I X } \\
\text { I X } \\
\text { I X }\end{array}$ & ley $\left(\Delta^{\mathrm{c}}\right.$ & $0 \rightarrow$ & & $\begin{array}{l}\mathrm{U}_{\text {crítico }} \\
\mathrm{U}_{\text {calcula }} \\
\mathrm{U}_{\text {calcul }} \\
\mathrm{U}_{\text {calcul }}\end{array}$ & $\begin{array}{l}=23,0 \\
=49 \\
=16 \\
=0\end{array}$ & & \multicolumn{3}{|c|}{$\begin{array}{l}(p=0,97) \\
(p<0,05)^{*} \\
(p<0,05)^{*}\end{array}$} & & & \\
\hline
\end{tabular}




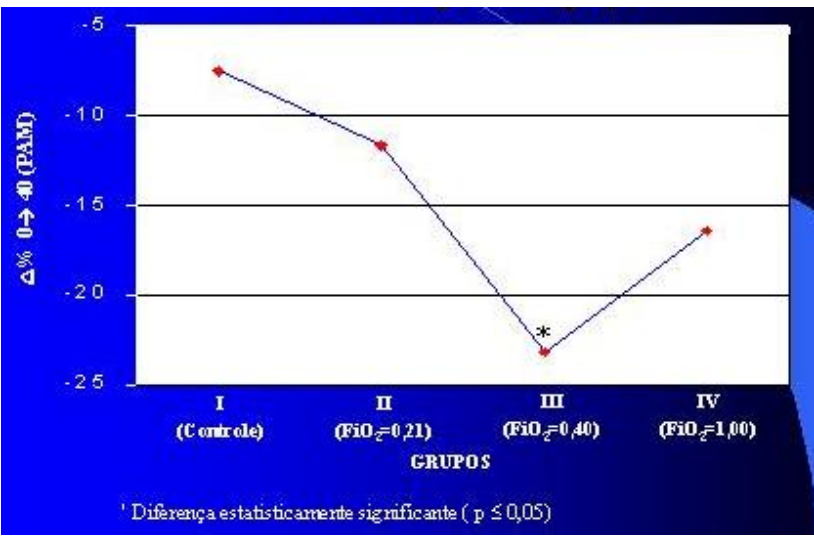

FIGURA 2 - Valores médios da pressão arterial média sistêmica (PAM), segundo os grupos.

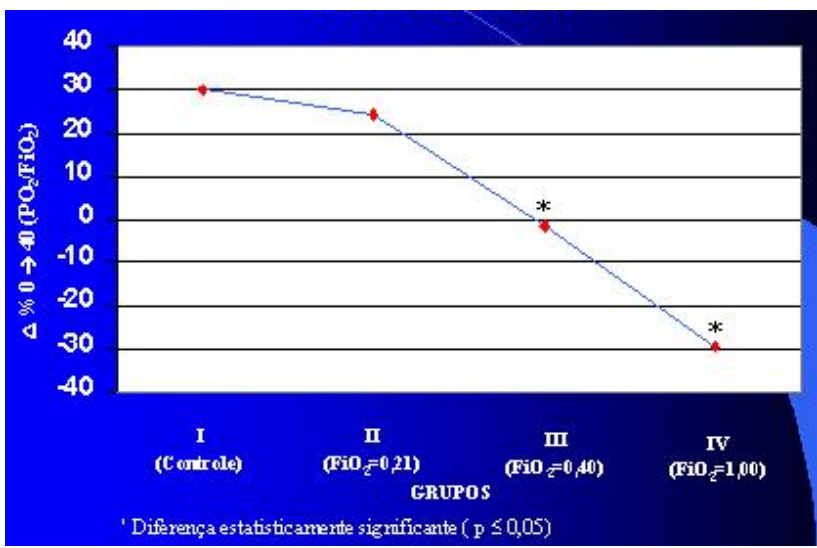

FIGURA 3 - Valores médios entre a pressão parcial de oxigênio arterial e a fração inspirada de oxigênio $\left(\mathrm{PO}_{2} /\right.$ $\mathrm{FiO}_{2}$ ), segundo os grupos.

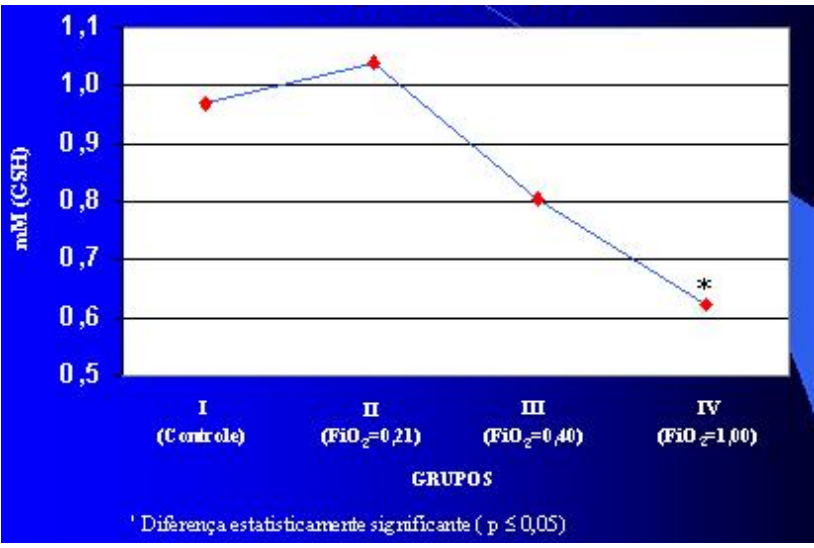

FIGURA 4 - Valores médios da glutationa reduzida nos pulmões submetidos à isquemia-reperfusão, segundo os grupos.

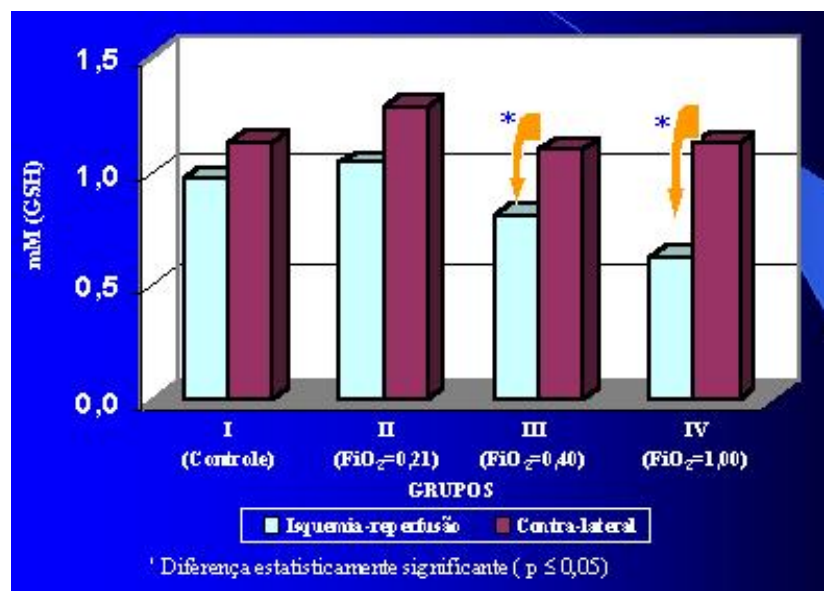

FIGURA 5 - Valores médios da glutationa reduzida nos pulmões submetidos à isquemia-reperfusão e contra-lateral dentro de cada o grupo.

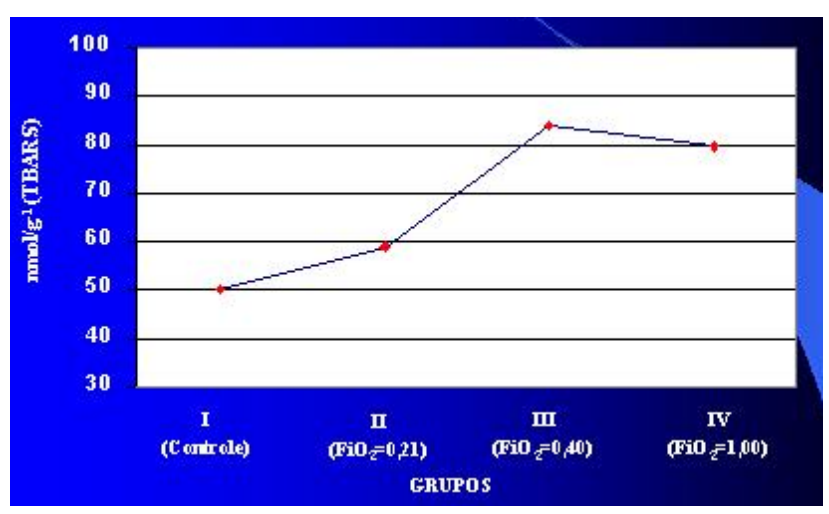

FIGURA 6 - Valores médios das substâncias reativas ao ácido tiobarbitúrio nos pulmões submetidos à isquemiareperfusão, segundo os grupos.

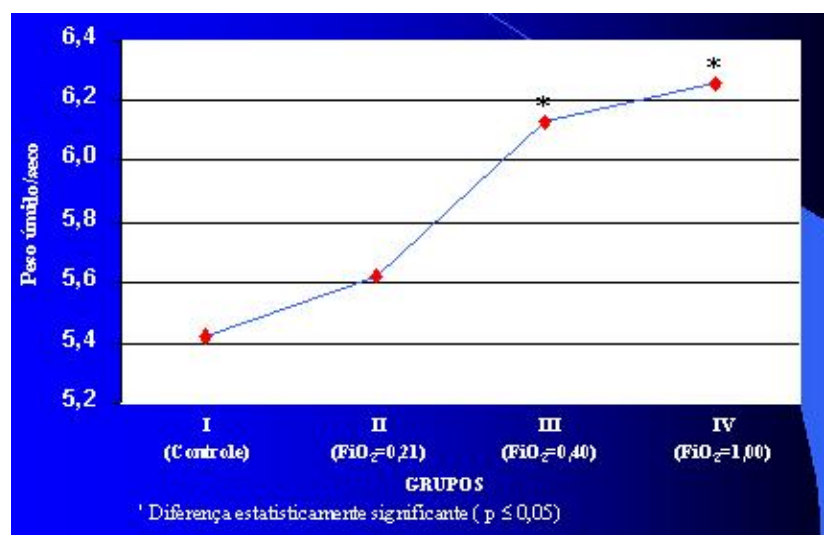

FIGURA 7 - Valores médios da relação entre o peso pulmonar úmido e o peso pulmonar seco, segundo os grupos. 
TABELA 3 - Valores da glutationa reduzida (GSH), em mM, no pulmão esquerdo (esq.) e direito (dir.), segundo os grupos.

\begin{tabular}{|c|c|c|c|c|}
\hline & Grupo I & Grupo II & Grupo III & Grupo IV \\
\hline & Esq. dir. & Esq. dir. & esq. dir. & Esq. dir. \\
\hline & $0,5430,564$ & $0,948 \quad 1,419$ & $0,748 \quad 1,150$ & $0,6701,346$ \\
\hline & $0,947 \quad 1,384$ & $0,8631,448$ & $0,720 \quad 0,959$ & $0,580 \quad 0,967$ \\
\hline & $1,0991,107$ & $1,5390,850$ & $0,8190,842$ & $0,6701,026$ \\
\hline & $1,164 \quad 0,746$ & $0,655 \quad 0,967$ & $0,830 \quad 1,150$ & $0,5381,097$ \\
\hline & $0,3041,303$ & $0,7601,099$ & $0,441 \quad 1,014$ & $0,320 \quad 1,314$ \\
\hline & $0,624 \quad 1,147$ & $0,855 \quad 1,287$ & $0,7681,042$ & $0,597 \quad 1,069$ \\
\hline & $1,3881,42$ & $1,1341,252$ & $0,663 \quad 0,831$ & $0,842 \quad 0,811$ \\
\hline & $1,0451,084$ & $1,279 \quad 1,404$ & $0,975 \quad 1,521$ & $0,546 \quad 0,858$ \\
\hline & $1,037 \quad 1,568$ & $0,9831,451$ & $0,827 \quad 1,131$ & $0,624 \quad 1,482$ \\
\hline & $1,560 \quad 0,971$ & $1,377 \quad 1,708$ & $1,2361,334$ & $0,815 \quad 1,267$ \\
\hline Média & $0,9701,129$ & $1,039 \quad 1,288$ & $0,8021,097$ & $0,6221,123$ \\
\hline
\end{tabular}

Kruskal-Wallis (I x II x III x IV) pulmão esquerdo: pulmão direito:

Mann-Whitney (pulmão esquerdo)

$$
\begin{aligned}
& \text { I x II } \\
& \text { I x III } \\
& \text { I x IV }
\end{aligned}
$$

Wilcoxon (esquerdo $\mathrm{X}$ direito)

$$
\begin{aligned}
& \text { I } \\
& \text { II } \\
& \text { III } \\
& \text { IV }
\end{aligned}
$$

\begin{tabular}{|c|c|c|c|c|c|c|c|c|}
\hline & Grup & & Grul & o II & Grup & III & Grup & IV \\
\hline & Esq. & dir. & esq. & dir. & esq. & dir. & esq. & \\
\hline & & 7 & 125 & 129 & 161 & 51 & 98 & 71 \\
\hline & 4 & 38 & 51 & 27 & 100 & 102 & 145 & 145 \\
\hline & 16 & 24 & 8 & 24 & 94 & 67 & 82 & 71 \\
\hline & 82 & 51 & 8 & 12 & 106 & 71 & 63 & 75 \\
\hline & 12 & 4 & 94 & 52 & 71 & 94 & 43 & 55 \\
\hline & 98 & 41 & 75 & 86 & 27 & 16 & 96 & 47 \\
\hline & 31 & 4 & 20 & 20 & 16 & 12 & 24 & 35 \\
\hline & 27 & 24 & 63 & 99 & 67 & 114 & 51 & 35 \\
\hline & 86 & 129 & 67 & 71 & 106 & 71 & 106 & 75 \\
\hline & 103 & 81 & 82 & 75 & 95 & 47 & 98 & 48 \\
\hline Média & 50 & 40 & 59 & 59 & 84 & 64 & 80 & 65 \\
\hline Kruska & $\begin{array}{l}\text { is }(\mathrm{Ix} \\
\text { áo esq } \\
\text { ăo dire }\end{array}$ & $\begin{array}{l}\text { II x III x IV) } \\
\text { lerdo: } \\
\text { ito: }\end{array}$ & & $\begin{array}{l}\chi_{\text {crítico }}^{2} \\
\chi_{\text {calcul }}^{2} \\
\chi_{\text {calcul }}^{2}\end{array}$ & & & $\begin{array}{l}(p=0,27) \\
(p=0,15)\end{array}$ & \\
\hline $\begin{array}{r}\text { Wilcoxc } \\
\text { I } \\
\text { I } \\
\text { II } \\
\text { IV }\end{array}$ & querd & X direito) & & $\begin{array}{l}\text { crítico }=8 \\
\text { calculado } \\
\Gamma \text { calculado } \\
\Gamma_{\text {calculado }} \\
\text { calculado }\end{array}$ & & & $\begin{array}{l}(p=0, \\
(p=) \\
(p=0, \\
(p=0,\end{array}$ & $\begin{array}{l}\text { 5) } \\
\text { 76) } \\
3) \\
9)\end{array}$ \\
\hline
\end{tabular}

$$
\begin{array}{ll}
\chi_{\text {crítico }}^{2}=7,82 & \\
\chi_{\text {calculado }}^{2}=12,57 & (\mathrm{p}<0,05)^{*} \\
\chi_{\text {calculado }}^{2}=3,15 & (\mathrm{p}=0,36) \\
\mathrm{U}_{\text {crítico }}=23,00 & \\
\mathrm{U}_{\text {calculado }}=48,00 & (\mathrm{p}=0,91) \\
\mathrm{U}_{\text {calculado }}=34,00 & (\mathrm{p}=0,24) \\
\mathrm{U}_{\text {calculado }}=22,50 & (\mathrm{p}<0,05)^{*}
\end{array}
$$

$$
\begin{aligned}
& \mathrm{T}_{\text {crítico }}=8,00 \\
& \mathrm{~T}_{\text {calculado }}=14,00 \\
& \mathrm{~T}_{\text {calculado }}=10,00 \\
& \mathrm{~T}_{\text {calculado }}=0,00 \\
& \mathrm{~T}_{\text {calculado }}=1,00
\end{aligned}
$$$$
\begin{aligned}
& (p=0,16) \\
& (p=0,07) \\
& (p<0,05)^{*} \\
& (p<0,05)^{*}
\end{aligned}
$$

TABELA 4 - Valores das TBARS, em nmol/g-1 , no pulmão esquerdo (esq.) e direito (dir.), segundo os grupos. 
TABELA 5 - Relação entre o peso pulmonar úmido e o peso pulmonar seco nos pulmões submetidos à isquemiareperfusão, segundo os grupos.

\begin{tabular}{|c|c|c|c|c|}
\hline & Grupo I & Grupo II & Grupo III & Grupo IV \\
\hline & 4,08 & 5,76 & 6,79 & 6,24 \\
\hline & 5,20 & 6,01 & 6,27 & 7,27 \\
\hline & 5,36 & 6,32 & 5,71 & 7,03 \\
\hline & 6,13 & 5,57 & 6,28 & 6,23 \\
\hline & 5,86 & 5,21 & 6,75 & 7,39 \\
\hline & 5,52 & 4,26 & 6,22 & 6,19 \\
\hline & 4,86 & 6,62 & 6,25 & 5,52 \\
\hline & 5,49 & 5,83 & 5,07 & 6,24 \\
\hline & 5,35 & 5,41 & 6,24 & 8,09 \\
\hline & 6,37 & 5,30 & 5,77 & 6,16 \\
\hline Média & 5,42 & 5,62 & 6,13 & 6,26 \\
\hline
\end{tabular}

Kruskal-Wallis (I x II x III x IV) pulmão esquerdo:

Mann-Whitney (pulmão esquerdo)

$$
\text { I x II }
$$

I $x$ III

I x IV

\section{Discussão}

A qualidade da preservação pulmonar é influenciada pelas alterações decorrentes da isquemia-reperfusão do órgão que interfere diretamente no resultado do transplante pulmonar.

Durante a isquemia, ocorre uma interrupção na oferta de oxigênio e nutrientes à célula, dificultando a manutenção do metabolismo celular aeróbio, o que resulta numa diminuição dos níveis de trifosfato de adenosina (ATP) intracelular ${ }^{17}$.

$O$ déficit de ATP altera o transporte ativo de íons através da membrana celular, levando a um excesso de sódio e cálcio no meio intracelular, o que resulta em edema e ativação de enzimas proteolíticas capazes de provocar a morte da célula 17.

Paralelamente, o metabolismo anaeróbio leva ao acúmulo de hipoxantina e à conversão da enzima xantina-desidrogenase em xantinaoxidase, no pulmão isquêmico. Ao ocorrer a reperfusão e a reintrodução do oxigênio molecular no metabolismo celular, a hipoxantina, por ação da xantina-oxidase, reage com esse oxigênio, liberando substâncias conhecidas como espécies reativas de oxigênio (EROs), que apresentam grande poder de lesão celular e tecidual ${ }^{18}$.

$$
\begin{aligned}
& (\mathrm{p}<0,05)^{*} \\
& (\mathrm{p}=0,48) \\
& (\mathrm{p}<0,05)^{*} \\
& (\mathrm{p}<0,05)^{*}
\end{aligned}
$$

Esse dano é conhecido como "lesão da isquemiareperfusão" e parece ser o fator que mais interfere na função pós-operatória imediata do transplante pulmonar.

No sentido de diminuir a lesão da isquemiareperfusão leva-se em consideração o estado da insuflação pulmonar onde a ventilação parece exercer efeitos benéficos. Do mesmo modo, a fração inspirada de oxigênio $\left(\mathrm{FiO}_{2}\right)$ também influencia na lesão da isquemia-reperfusão, não estando a $\mathrm{FiO}_{2}$ ideal perfeitamente determinada.

Com o objetivo de avaliar a ventilação com as $\mathrm{FiO}_{2}$ a 0,21, 0,40 e 1,00, na isquemia-reperfusão pulmonar, foi idealizado o presente estudo. Foram utilizados como parâmetros de avaliação a medida da pressão arterial média sistêmica (PAM), a relação entre a pressão parcial de oxigênio arterial e a fração inspirada de oxigênio $\left(\mathrm{PO}_{2} / \mathrm{FiO}_{2}\right)$, as dosagens teciduais da glutationa reduzida (GSH) e das substâncias reativas ao ácido tiobarbitúrico (TBARS) e a relação entre o peso pulmonar úmido e o peso pulmonar seco.

A opção por esses parâmetros foi feita no sentido de avaliar possíveis repercussões em nível local e/ou sistêmico. 
O tempo de isquemia foi de 30 minutos, e levou em consideração os trabalhos de Buchanan e col. ${ }^{19}$ e Castro e col. ${ }^{20}$ os quais mostraram que esse tempo, em condições de normotermia, detecta lesões celulares decorrentes da isquemia tecidual.

O tempo de reperfusão baseou-se nos trabalhos de Sakamoto e col. ${ }^{21}$, De Campos e col. ${ }^{22}$ e Halldorsson e col. ${ }^{23}$, que observaram manifestações da lesão da isquemia-reperfusão após 10 minutos de reperfusão pulmonar.

A medida da pressão arterial média sistêmica (PAM) foi realizada com o objetivo de avaliar possíveis efeitos sistêmicos da isquemiareperfusão pulmonar.

O metabolismo anaeróbio promove o acúmulo de metabólitos tóxicos no tecido isquêmico, que seriam liberados para a circulação sangüínea sistêmica, após a reperfusão, levando à acidose metabólica, hipercalemia, mioglobinúria, insuficiência renal e hipotensão arterial. Segundo Haimovici $^{24}$, a intensidade dessas manifestações seria proporcional à quantidade de substâncias liberadas.

No presente estudo, os grupos submetidos à isquemia-reperfusão pulmonar mostraram uma diminuição da PAM em relação ao grupo-controle, sendo mais acentuada nos grupos ventilados com elevadas $\mathrm{FiO}_{2}$ (40\% e 100\%), e significante no grupo ventilado com oxigênio a $40 \%$.

A ventilação com elevadas $\mathrm{FiO}_{2}$, durante a isquemia-reperfusão pulmonar, tem sido relacionada a uma maior peroxidação lipídica da membrana celular, o que liberaria ácido aracdônico e, conseqüentemente, levaria à formação de prostaglandinas ${ }^{25}$. Essas substâncias, apresentando propriedades vasodilatadoras, associar-se-iam aos metabólitos tóxicos formados durante a isquemia e explicariam a maior diminuição da PAM nos grupos ventilados com oxigênio a $40 \%$ e $100 \%$.

No grupo ventilado com oxigênio a $21 \%$, durante a isquemia-reperfusão, observou-se uma menor diminuição da PAM, o que sugere uma menor lesão celular com essa ventilação.

A pressão parcial de oxigênio arterial $\left(\mathrm{PO}_{2}\right)$ constitui um dos principais parâmetros de avaliação da isquemia-reperfusão pulmonar, porque reflete de maneira global as lesões decorrentes desse período.
A análise da relação $\mathrm{PO}_{2} / \mathrm{FiO}_{2}$, como método de avaliação da função pulmonar, foi realizada para permitir a comparação entre os grupos e diminuir a possibilidade de que as diferenças encontradas fossem determinadas pelo padrão ventilatório.

A função pulmonar depende diretamente da integridade da membrana celular da rede alvéolocapilar do pulmão, cuja estrutura é particularmente sensível aos fatores que envolvem a isquemiareperfusão, como a diminuição dos níveis energéticos celulares e a ação de EROs ${ }^{26}$.

Os resultados do presente estudo mostraram que não houve diferença significante na relação $\mathrm{PO}_{2} / \mathrm{FiO}_{2}$ entre o grupo controle e o grupo ventilado com oxigênio a $21 \%$, enquanto nos grupos ventilados com oxigênio a $40 \%$ e $100 \%$ ocorreu uma diminuição significante dessa relação.

Esses resultados sugerem benefícios da ventilação pulmonar com baixa $\mathrm{FiO}_{2}$. Isso seria devido a uma oferta constante de oxigênio no alvéolo, em baixas concentrações, que contribuiria para a manutenção do metabolismo celular aeróbio e uma menor liberação de EROs.

Resultados semelhantes foram observados por Bhabra e col. ${ }^{27}$, que mostraram uma melhor relação da $\mathrm{PO}_{2} / \mathrm{FiO}_{2}$ em pulmões ventilados com oxigênio a $20 \%$, quando comparados a pulmões ventilados com oxigênio a $100 \%$, num modelo de isquemiareperfusão pulmonar hipotérmica em ratos.

A possível liberação de EROs foi avaliada por meio da dosagem tecidual da glutationa reduzida (GSH) e das substâncias reativas ao ácido tiobarbitúrico (TBARS).

A GSH representa o principal antioxidante não-enzimático hidrossolúvel da célula e exerce seus efeitos desintoxicantes ao reagir com o peróxido de hidrogênio e outros peróxidos, transformando-se na sua forma oxidada (GSSG). Desse modo, ela contribui para a manutenção da normalidade celular, uma vez que os peróxidos, que são EROs, apresentam um alto poder lesivo à célula ${ }^{28}$.

Soncul e col. ${ }^{29}$ mostraram uma diminuição nos níveis teciduais de GSH, após um período de isquemia pulmonar normotérmica, e relacionaram esse fato a um consumo desse antioxidante na neutralização de EROs. 
No presente estudo, os valores da GSH mostraram uma diminuição linear com o aumento da $\mathrm{FiO}_{2}$, nos grupos submetidos à isquemiareperfusão, quando comparados com o grupocontrole, sendo significante no grupo ventilado com oxigênio a 100\%.

Quando se compararam os valores da GSH no pulmão contra-lateral, nos quatro grupos entre si, não se observaram diferenças significantes entre eles. Isso denota uma ausência ou baixa formação de EROs no pulmão contra-lateral.

Quando se compararam os valores da GSH dentro de cada grupo, ocorreu uma diminuição significante entre o pulmão submetido à isquemiareperfusão e o pulmão contra-lateral, nos grupos ventilados com oxigênio a $40 \%$ e $100 \%$ e sem diferença significante nos grupos controle e ventilado com oxigênio a $21 \%$.

Esses achados corroboram o maior consumo da GSH nos grupos submetidos à isquemiareperfusão com elevadas $\mathrm{FiO}_{2}$, sugerindo uma maior formação de EROs nesses grupos. Como nos grupos controle e ventilado com oxigênio a $21 \%$ não houve diferenças significantes nos valores da GSH, infere-se que a ventilação com oxigênio a 21\% levou a uma menor produção de EROs.

As substâncias reativas ao ácido tiobarbirtúrico (TBARS) correspondem a metabólitos originados pela destruição da membrana celular em conseqüência da ação lesiva de EROs sobre essa estrutura celular e avaliam de modo quantitativo a lesão oxidativa ${ }^{30}$.

Entre as EROs, destaca-se o radical hidroxila, formado pela reação do ânion superóxido com o peróxido de hidrogênio, por meio da reação de Fenton, constituindo-se num dos metabólitos mais lesivos à célula ${ }^{31}$.

Esse radical apresenta uma grande afinidade por lipídios e proteínas da membrana celular, o que provoca uma reação em cadeia com alto poder de destruição celular que se refletirá num aumento dos níveis de TBARS.

Os resultados obtidos, nesse estudo, mostraram uma tendência de aumento nos valores das TBARS nos grupos submetidos à isquemiareperfusão ventilados com $\mathrm{FiO}_{2}$ elevadas, sugerindo uma maior formação de EROs nesses grupos, quando comparadas à ventilação com baixa $\mathrm{FiO}_{2}$.

Do mesmo modo, Fisher e col. ${ }^{32}$, estudando a influência da $\mathrm{FiO}_{2}$ na isquemia-reperfusão pulmonar, mostraram que a ventilação com oxigênio a $50 \%$ ou $95 \%$, quando comparada com a ventilação com oxigênio a $5 \%$ ou $20 \%$, apresentou maiores níveis de TBARS, sugerindo maior peroxidação lipídica com altas $\mathrm{FiO}_{2}$. Sugeriram também que esse fenômeno teria seu início já na fase de isquemia.

A relação entre o peso pulmonar úmido e o peso pulmonar seco reflete o edema pulmonar decorrente da isquemia-reperfusão. Bleiwess e $\mathrm{col}^{33}$ observaram uma relação entre o coeficiente de filtração capilar e a relação entre o peso pulmonar úmido e o peso pulmonar seco, num modelo de isquemia-reperfusão pulmonar normotérmica em ratos.

Esse edema pulmonar resultaria de uma disfunção do endotélio vascular e seria mediado, principalmente, pela liberação de EROs e por um desequilíbrio entre substâncias vasodilatadoras e vasoconstritoras ${ }^{33}$.

O predomínio de substâncias vasoconstritoras atuaria nas propriedades contráteis das células endoteliais e, com isso, alteraria as funções da barreira endotelial, resultando num aumento da permeabilidade capilar. Essa ação parece ser mediada pelos níveis celulares de adenosinamonofostato cíclico (AMPc) e guanosinamonofosfato cíclico (GMPc) ${ }^{34}$.

Esses fatos têm justificado a utilização de substâncias, como a prostaglandina $\mathrm{E}_{1}\left(\mathrm{PGE}_{1}\right)$ e o óxido nítrico (NO), no intuito de diminuir a lesão da isquemia-reperfusão. Essas substâncias, além de produzirem vasodilatação, participariam na redução da agregação leucocitária e plaquetária e na supressão do fator de necrose tumoral ${ }^{35}$.

O resultado desse experimento mostrou um aumento progressivo na relação entre o peso pulmonar úmido e o peso pulmonar seco, nos grupos submetidos à isquemia-reperfusão, de modo linear com o aumento da $\mathrm{FiO}_{2}$, sendo significante nos grupos ventilados com oxigênio a $40 \%$ e $100 \%$.

Provavelmente, isso se deveu a uma maior ação de EROs nesses grupos que, além de causarem lesão celular direta, estimularam a produção de mediadores inflamatórios, 
contribuindo para um aumento da permeabilidade capilar e formação do edema pulmonar.

Esses dados corroboram os de Fisher e col. ${ }^{32}$ e Bleiwess e col. ${ }^{33}$, que mostraram uma diminuição do edema pulmonar, decorrente da isquemiareperfusão, nos pulmões ventilados com $\mathrm{FiO}_{2}$ a 0,20 .

Numa avaliação global, os resultados sugeriram que a ventilação pulmonar com $\mathrm{FiO}_{2}$ a 0,21 correlacionou-se com uma menor lesão decorrente da isquemia-reperfusão, quando comparada àquelas realizadas com $\mathrm{FiO}_{2}$ mais elevadas (o,40 e 1,00) na isquemia-reperfusão pulmonar.

\section{Conclusão}

A ventilação com fração inspirada de oxigênio a 0,21 , quando comparada à ventilação com frações inspiradas de oxigênio a 0,40 e 1,00, durante o período de isquemia-reperfusão pulmonar normotérmica, mostrou uma menor diminuição da pressão arterial média sistêmica, uma melhor relação entre a pressão parcial de oxigênio arterial e a fração inspirada de oxigênio, maiores valores nas medidas da glutationa reduzida, uma menor produção de substâncias reativas ao ácido tiobarbitúrico e uma menor formação de edema pulmonar.

\section{Referências}

1. Cooper JD, Ginsberg RJ, Goldberg M, Patterson GA, Pearson FG, Todd TRJ. Unilateral lung transplantation for pulmonary fibrosis. N Engl J Med 1986;314:1140-5.

2. Meyers BF, Patterson GA. Lung transplantation: current status and future prospects. World J Surg 1999;23:115662.

3. Khan SU, Salloum J, O’Donovan PB, Mascha EJ, Metha AC, Matthay MA. Acute pulmonary edema after lung transplantation. Chest 1999;116:187-94.

4. Unruh HW. Lung preservation and lung injury. Chest Surg Clin N Am 1995;5:91-106.

5. Akashi A, Nakahara K, Kamiike W, Matsumura A, Hatanaka N, Kawashima Y. Attenuation of warm ischemic injury of rat lung by insuflation with room air: assesment of cellular components and the surfactant in the bronchoalveolar lavage fluid in relation to changes in cellular adenosine triphosphate. Transplantation 1993;55:24-30.

6. Husen B, Ramsamooj R, Hewitt CW, Schroder F, Beuke $\mathrm{M}$, Poets CF. The importance of static lung inflation during organ storage. Transplantation 1996;62: 1720-5.

7. Ulicny KS Jr, Egan TM, Lambert CJ Jr, Reddick RL, Wilcox BR. Cadaver lung donors: effect of preharvest ventilation on graft function. Ann Thorac Surg 1993;55:1185-91.

8. Cardoso JJD. Efeito da ventilação na isquemia-reperfusão pulmonar em ratos [Tese]. São Paulo: Universidade Federal de São Paulo - Escola Paulista de Medicina; 1998.

9. D’Armini AM, Lemasters JJ, Egan TM. Studies of rat lung viability and adenine nucleotide metabolism after death. Ann Thorac Surg 1996;62:1448-53.

10. Koyama I, Toung TJK, Gurtner GH, Traystman RJ. $\mathrm{O}_{2}$ radicals mediate reperfusion lung injury in ischemic $\mathrm{O}_{2}-$ ventilated canine pulmonary lobe. J Appl Physiol 1987;63:111-5.

11. Van Raemdonck DEM, Jannis NCP, Rega FRL, De Leyn PRJ, Flameng WJ, Lerut TE. Delay of adenosine triphosphate depletion and hypoxanthine formation in rabbit lung after death. Ann Thorac Surg 1996;62:23341.

12. Hamvas A, Park Cpalazzo R, Liptay M, Cooper J, Schuster DP. Modifyng pulmonary ischemia-reperfusion injury by altering ventilatory strategies during ischemia. J Appl Physiol 1992;73:2112-9.

13. Hennington MH, D’Armini AM, Lemasters JJ, Egan TM. Cadavers lungs for transplantation. Transplantation 1996;61:1009-14.

14. Zhao G, Al-Medhi AB, Fisher AB. Anoxiareoxynenation versus ischemia in isolated rat lungs. Am J Physiol 1997;273:1112-7.

15. Bleuter E., Robson, MJ, Butternweser E. The glutathione instability of drug sensitive red ceclls. J Lab Clin Med 1957;49:84-95.

16. Siegel S. Estatística não-paramétrica. São Paulo: Ed. McGraw-Hill; 1975.

17. Pinheiro BV. Lesão pulmonar de reperfusão. J Pneumol 1999;25:124-36.

18. McCord JM. Oxygen-derived free radicals in postischemic tissue injury. N Engl J Med 1985;312:15963.

19.Buchanan AS, DeLima NF, Binns OAR, Mauney MC, Cope JT, Langenburg SE. Pulmonary function after nonheart beating lung donation in survival model. Ann Thorac Surg 1995;60:38-46.

20. Castro MAA, Madruga GSP, Silva UL, Bock P, Klein AB, Felicetti JC. Quantificação dos produtos de peroxidação lipídica em modelo experimental de injúria de reperfusão pulmonar em ratos. J Pneumol 1996;22:65-8.

21. Sakamoto T, Yamashita C, Okada M. Efficacy of initial controlled perfusion pressure for ischemia-reperfusion injury in a 24-hour preserved lung. Ann Thorac Cardiovasc Surg 1999;5:21-6.

22. DeCampos KN, Keshavjee S, Slutsky AS, Liu M. Alveolar recruitment prevents rapid-reperfusioninduced injury of lung transplants. J Heart Lung Transplant 1999;18:1096-102.

23. Halldorsson AO, Kronon MT, Allen BS, Rahman S, 
Wang T. Lowering reperfusion pressure reduces the injury after pulmonary ischemia. Ann Thorac Surg 2000;69:198-204.

24. Haimovici M. Muscular, renal and metabolic complications of acute arterial oclusions. Surgery 1979;85:461-8.

25. Tanita T, Song C, Kubo H, Hoshikawa Y, Ueda S, Fujimura S. Superoxide possibly produced in endothelial cells mediates the neutrophil-induced lung injury. Ann Thorac Surg 2000;69:402-8.

26. Al-Mehdi AB, Zhao G, Fisher AB. ATP-independent membrane depolarization with ischemia in the oxygenventilated isolated rat lung. Am J Respir Cell Mol Biol 1998;18:653-61.

27. Bhabra MS, Hopkinson DN, Shaw TE, Hooper TL. Modulation of lung reperfusion injury by nitric oxide. Transplantation 1999;68:1238-43.

28. Bryan CL, Cohen DJ, Dew JA, Trinkle JK, Jenkinson SG. Glutathione decreases the pulmonary reimplantation response in canine lung autotransplants. Chest 1991;100:1694-702.

29. Soncul H, Oz E, Kalaycioglu S. Role of ischemic preconditiong on ischemia-reperfusion injury of the lung. Chest 1999;115:1672-7.
30. Sakamak F, Hoffmann H, Muller C, Dienemann H, Messmer K, Schildberg FW. Reduced lipid peroxidation and ischemia-reperfusion injury after lung transplantation using low potassium dextran solution for lung presevation. Am J Respir Crit Care Med 1997;156:1073-81.

31. Zhao G, Ayene IS, Fisher AB. Role of iron in ischemiareperfusion oxidative injury of rat lungs. Am J Respir Cell Mol Biol 1997;16:293-9.

32. Fisher AB, Dodia C, Tan Z, Ayene I, Eckenhoff RG. Oxygen-dependent lipid peroxidation during lung ischemia. J Clin Invest 1991;88:674-9.

33. Bleiweis MS, Jones DR, Hoffmann SC, Becker RM, Egan TM. Reduced ischemia-reperfusion injury with rolipram in rat cadaver lung donor: effect of cyclic adenosine monophosphate. Ann Thorac Surg 1999;67:194-200.

34. Miller DL, Roberts AM. Pulmonary artery occlusion and reperfusion causes microvascular constriction in the rabbit lung. Ann Thorac Surg 1999;67:323-8.

35. Lindberg L, Sjoberg T, Ingemansson R, Steen S. Inhalation of nitric oxide after lung transplantation. Ann Thorac Surg 1996;61:956-62.

\section{Correspondência}

Rafael José Silveira

Hospital Nereu Ramos

Rua Rui Barbosa, 800

88025-301 Florianópolis - SC

Tel/Fax: (48)228-4633

toracica@matrix.com.br

Recebimento: 11/06/2004

Revisão: 21/07/2004

Aprovação: 28/08/2004

Conflito de interesse: nenhum

Fonte de financiamento: nenhuma

\section{Como citar este artigo:}

Silveira RJ, Silva FM, Wilhelm Filho D, Cardoso JJD, Leão LEV. Estudo da fração inspirada de oxigênio na isquemiareperfusão pulmonar em ratos. Acta Cir Bras [serial online] 2004 Set-Out;19(5). Disponível em URL: http://www.scielo.br/acb [também em CD-ROM].

*Figura colorida disponível em www.scielo.br/acb 